\title{
Self-consistent inclusion of classical large-angle Coulomb collisions in plasma Monte Carlo simulations
}

\author{
A. E. Turrell ${ }^{1}$, M. Sherlock, S. J. Rose \\ Blackett Laboratory, Imperial College London, South Kensington, SW7 $2 A Z$
}

\begin{abstract}
Large-angle Coulomb collisions allow for the exchange of a significant proportion of the energy of a particle in a single collision, but are not included in models of plasmas based on fluids, the Vlasov-Fokker-Planck equation, or currently available plasma Monte Carlo techniques. Their unique effects include the creation of fast 'knockon' ions, which may be more likely to undergo certain reactions, and distortions to ion distribution functions relative to what is predicted by small-angle collision only theories. We present a computational method which uses Monte Carlo techniques to include the effects of large-angle Coulomb collisions in plasmas and which selfconsistently evolves distribution functions according to the creation of knock-on ions of any generation. The method is used to demonstrate ion distribution function distortions in an inertial confinement fusion ( $\mathrm{ICF}$ ) relevant scenario of the slowing of fusion products.
\end{abstract}

Keywords: Monte Carlo, large-angle collisions, knock-ons, reaction-in-flight, inertial confinement fusion, plasma

\section{Introduction}

Large-angle Coulomb collisions affect the distribution of energy in plasmas by allowing the transfer of a significant proportion of the energy of a particle in a single collision. It is well known that their importance relative to the small-angle Coulomb collisions which dominate interactions in classical plasmas is $\mathcal{O}(1 / \ln \Lambda)$ [1], where $\ln \Lambda$ is the Coulomb logarithm.

Their effects are therefore expected to be important in the $2 \lesssim \ln \Lambda \lesssim 5$ regime, which includes high intensity laser-plasma interactions at solid density [2], degenerate

\footnotetext{
${ }^{1}$ Corresponding author: a.turrell09@imperial.ac.uk
} 
plasmas [3], and stellar cores [4, 5]. This regime also includes inertial confinement fusion (ICF) [6, 7], as an igniting ICF hotspot will have conditions [8] of areal density and temperature of $\sim 0.3 \mathrm{~g} / \mathrm{cm}^{2}$ and $\sim 10 \mathrm{keV}$ respectively, which correspond to small values of $\ln \Lambda$ for a typical hotspot radius of tens of microns.

Wide ranging experimentally detectable consequences of large-angle collisions have been described; for the shape and evolution of distribution functions [9, 10, 11, 12], for fusion reactivities or as a diagnostic in both ICF and magnetic confinement fusion $(\mathrm{MCF})$ [13, 14, 15, 16, 17, 18, 19, 20, for plasma properties such as particle stopping and temperature equilibration [21, 22, 23], and for 'athermal' fusion [24, 25]. Non-Maxwellian distributions caused by large-angle collisions have been experimentally observed on JET (the Joint European Torus) [26].

A consequence of the inclusion of large-angle collisions is the generation of high energy 'knock-on' ions; these are fast particles generated by collisions between high energy fusion reaction products and thermal ions in the plasma in which the thermal ions can gain many times their initial kinetic energy, distorting the fuel ion distributions from thermal equilibrium in the process. The knock-ons may be more likely to fuse themselves (in what are known as reaction-in-flight fusion events), and can go on to generate further knock-ons. These processes must be fully understood in order to use Coulomb collision-induced knock-ons as a diagnostic.

The inclusion of the effects of large-angle Coulomb collisions in plasmas is a longstanding challenge [1, 27, 28], and we present a new method which applies to plasmas with $\ln \Lambda \gtrsim 2$. Although there are other methods which can include the effects of large-angle collisions and knock-ons, the approach presented has unique strengths as it self-consistently evolves ion distribution functions under the influence of largeangle collisions, but is less computationally intensive than molecular dynamics (MD) simulations.

Large-angle collisions involve a large transfer of energy or momentum per collision, as opposed to small-angle collisions. These two types of collision are also known as 'close' and 'remote' collisions respectively due to the relationship between impact parameter, $b$, and scattering angle, $\theta$, of $b=b_{\perp} \cot \left(\frac{\theta}{2}\right)$. Here, $b_{\perp}=\frac{q_{i} q_{j}}{4 \pi \epsilon_{0}} \frac{1}{m_{i j} v_{i j}^{2}}$ and $q$ is the charge, $m_{i j}$ is the reduced mass, $v_{i j}$ is the relative velocity, and the species are denoted by $i$ and $j$.

The most commonly used plasma theories are based on the Vlasov-Fokker-Planck (VFP) equation, and are only applicable in classical plasmas with $\ln \Lambda \gg 1$ as they either ignore large-angle Coulomb collisions [12, approximate them by over-counting the effects of small-angle collisions in $\ln \Lambda$ [27], or have to be expanded to higher orders to recover some of their effects [29, 22]. Current computational methods invoke the small-angle approximation [30, 31, 32, 33], and are not applicable to 
large-angle collisions.

Though small-angle collisions are important for the overall exchange of energy in plasmas with $2 \lesssim \ln \Lambda \lesssim 5$, the impact of fewer large-angle collisions with larger transfers of energy per collision can distort distribution functions. This can indirectly change the rate of exchange of energy between two plasma species as the rate of energy transfer is dependent upon their distribution functions. That the changes in energy per collision are discontinuous is also important; continuous loss models fail for large-angle scattering [34]. These problems typically manifest when one species has a much higher average energy than another, as is the case with the slowing down of alpha particles during burn in fusion plasmas.

Analytical theories which include the effects of large-angle collisions via the Coulomb logarithm for plasmas in thermal equilibrium have been developed, including those of Baalrud [35], Brown, Preston, and Singleton (BPS) [36], and Gericke, Murillo, and Schlanges [37]. These have been benchmarked against MD simulations [38, 39, 40], and produce the correct results for properties such as temperature equilibration [41]. The MD simulations used to benchmark these theories do not include quantum mechanical effects $a b$ initio, so electron interactions are usually modified at short distances [42, 39, 43, 41] to prevent them becoming infinitely bound to ions. However, Dimonte and Daligault [38] presented purely classical results, using only the assumption of like charges in a neutralising background, and found agreement with the classical BPS theory for temperature equilibration [36]. This is strong evidence that these analytical theories are including the effects of classical ion-ion large-angle collisions. However, they involve an integration over assumed Maxwell-Boltzmann distribution functions, and are not designed to give any information about the creation of knock-ons due to large-angle collisions, or the distortion to ion distribution functions caused by them.

Several semi-analytical approaches to calculating the effects of large-angle collisions for non-thermal distributions have been developed. In one, which shall be referred to as the Ryutov theory, the Rutherford cross-section is directly integrated, and a source term for knock-on ions found [10, 44, 19, 16]. This requires assumptions about the initial ion distribution functions, and uses a cut-off in impact parameter between close and remote collisions which is either a multiple of $b_{\perp}$, or is imposed by computational limits. An initial non-Maxwellian fuel ion distribution is calculated from the knock-on source term, and is subsequently evolved according to a smallangle-only VFP equation. Knock-ons of higher order are ignored, so the Ryutov theory only includes the first generation of knock-on ions. Other semi-analytical approaches only model the athermal part of the distribution function. This approach is usually limited by the assumption that there is no feedback on the bulk distribution 
function [24], or by the assumption of a steady state athermal distribution [25].

MD simulations are able to model any distribution function, and can resolve the hard collisions which are responsible for knock-on ions. Though MD calculations make few assumptions, they are computationally intensive and typically use a maximum of tens of thousands of particles [39, 45]. We find that millions of simulation particles are required to resolve the distortion to the tails of ion distribution functions caused by large-angle Coulomb collisions, and that an alternative method of calculation of their effects, adopting a Coulomb logarithm benchmarked by MD simulations, is therefore desirable.

In the Monte Carlo based method developed here, a cut-off in impact parameter is used to separate out large- and small-angle Coulomb collisions and all ion-ion interactions have a chance of generating knock-ons. The rate of generation of knockon ions is benchmarked against Ryutov's theory.

The strength of the approach presented is that it includes knock-ons of any generation, and it allows distribution functions to evolve self-consistently according to those knock-ons and to other changes due to fusion reactions. As a demonstration of the application of this method, the effects of the distortions to ion distribution functions on the source neutron spectra of the hotspot in a burning ICF capsule are calculated.

Large-angle collisions between electrons and ions, and electrons and electrons, are omitted from the analysis presented as the dynamics of these interactions are modified at the short ranges relevant to large-angle scattering by quantum mechanical effects [46, 47]. These effects would not be included by the classical approach presented. Furthermore, the large mass difference between electrons and ions means both that energy exchange between electrons and ions is much less effective than between different species of ion, and that electrons are less likely to maintain a nonMaxwellian distribution function for timescales long enough to significantly change the evolution of plasmas for which this method is likely to be useful.

\section{Theory of large-angle Coulomb collisions}

We take large-angle collisions to be true binary collisions, where the full postcollision trajectory is followed according to the Rutherford cross-section. This is different to 'multiple' small-angle scattering in which a particle is deflected due to an ensemble of particles within its Debye sphere (a sphere of radius the plasma Debye length $\lambda_{\mathrm{D}}$ ). Multiple small-angle scattering is described using the binary collision $a p$ proximation, but particles undergoing this scattering do not follow the post-collision trajectory of two particles interacting with the Rutherford cross-section. There are 
two equivalent pictures of the origin of large-angle, and multiple small-angle, collisions.

In one, described by Cohen, Spitzer, and McRoutly [27], and others [1, 48], it is the long range of the Coulomb force which causes remote particles to dominate interactions in classical plasmas. These long-range forces are cut off at $b=\lambda_{\mathrm{D}}$ in order to avoid the divergence in the cross-section. However, they note that there must be some finite distance $b_{c} \leq \lambda_{\mathrm{D}}$ where the interaction with one other particle becomes dominant, that if there were no large deflections then plasmas would be fully described by VFP, and that the omission of large-angle encounters introduces a larger error as $\lambda_{\mathrm{D}} / b_{\perp}$ and $\ln \Lambda$ become smaller. The other view, from Sivukhin [28], is that the divergence arises because Rutherford implicitly assumes infinite interaction times, but the time for remote interactions must be constrained. This is because deflection angles in remote collisions must both be small, and increase with interaction time. Large-angle collisions are effectively instantaneous by comparison, thus particles follow the 'full' Rutherford collision trajectory.

These authors that we refer to [27, 1, 48, 28] recommend approximating largeangle collisions by using $b_{c}=0$ in the absence of simple methods to include a $b_{c}>0$, with Sivukhin adding that "Such an approach... cannot pretend to give a completely adequate description of the true situation." Both interpretations suggest the use of an impact parameter $b=b_{c}$ which defines the cross-over between large- and smallangle scattering, so that the former is restricted in angle to $\left|\theta_{c}\left(b_{c}\right)\right| \leq|\theta| \leq \pi$, and the latter to $0 \leq|\theta|<\left|\theta_{c}\left(b_{c}\right)\right|$.

Previous work has examined the evolution of plasmas under the influence of largeangle collisions and with $b_{c}>0$. Values for $b_{c}$ used include $b_{\perp}, 2 b_{\perp}$ [1], 5.67b $b_{\perp}$ [16], and values set by computational limits [24]. Given that the parameter $b_{c}$ is unknown, three representative values of it are adopted in order to examine the effects of varying it. The choice adopted by many authors of $b_{c}=b_{\perp}$ is included, and corresponds to a cut-off in angle of $\theta_{c}=\pi / 2$. The value of the cut-off equivalent to considering smallangle collisions only is also used in simulations, and is given by $b_{c}=0$. If $b_{c}=0$, no large-angle collisions are included. Finally, the value of $b_{c}=3 b_{\perp}$ is used in order to provide an approximate comparison with theories which take a much smaller angle than $\theta_{c}=\pi / 2$ to be the cross-over angle between large- and small-angle scattering [16]. These values of $b_{c}$ are used to model the strength of the large-angle collisions in our combined large- and small-angle collision algorithm. $b_{c}$ plays its most important role in equations (8), (9), and (10) where it causes the reduction in the strength of small-angle collisions, appears in the cross-section for large-angle collisions, and is used to generate large-angle scattering angles respectively. The correct value of $b_{c}$ remains an open question, and is not addressed here. Too large a cut-off is not 
consistent with the fact that stopping powers are dominated by small-angle collisions, and no cut-off is not consistent with the well-known phenomenon of Coulomb-induced knock-on ions, meaning that the true situation is likely to be represented by some $\lambda_{\mathrm{D}} \gg b_{c}>0$.

\section{Algorithm including small- and large-angle Coulomb collisions}

\subsection{Plasma simulations with small-angle collisions only}

We begin with a brief review of Monte Carlo based techniques that simulate the evolution of part or all of a plasma under the influence of multiple, small-angle collisions. These include those by Takizuka and Abe (TA) [30], Nanbu [31], and Sherlock 32. They share a similar approach [33, being based on small-angle approximations of the Boltzmann equation. The Boltzmann equation for interaction of species $i$ is

$$
\frac{\partial f_{i}}{\partial t}+\mathbf{v}_{i} \cdot \nabla_{x} f_{i}+\frac{\mathbf{F}}{m_{i}} \cdot \nabla_{v} f_{i}=\sum_{j} C_{B}\left(f_{i}, f_{j}\right)
$$

with

$$
C_{B}\left(f_{i}, f_{j}\right)=\int_{\mathbb{R}^{3}} \mathrm{~d} v_{j} \int_{S^{2}} \mathrm{~d} \Omega\left(\frac{\mathrm{d} \sigma}{\mathrm{d} \Omega}\left(v_{i j}, \Omega\right)\right)\left(f_{i}^{\prime} f_{j}^{\prime}-f_{i} f_{j}\right)
$$

the Boltzmann collision term, primes denoting post-collision distributions, and where $\frac{\mathrm{d} \sigma}{\mathrm{d} \Omega}$ is, for a classical plasma, the pure Rutherford cross-section given by

$$
\frac{\mathrm{d} \sigma}{\mathrm{d} \Omega}=\left(\frac{q_{i} q_{j}}{4 \pi \epsilon_{0}}\right)^{2} \frac{1}{m_{i j}^{2} v_{i j}^{4}} \frac{1}{4 \sin ^{4} \frac{\theta}{2}}
$$

The force term $\mathbf{F}$ hides a lot of complexity; it must include $\mathbf{E}$ and $\mathbf{B}$ fields both from external sources and from plasma motion, and those fields must also satisfy Maxwell's equations. The most important plasma interactions are dominated by those at long distances and small deflection angles, and which therefore produce little change in velocity in each collision. To provide computational methods for simulating plasmas, it is usual to Taylor expand (1) in $\Delta \mathbf{v}$ (the change in velocity in a single collision) to give the small-angle only Landau approximation of the Boltzmann equation. This results in a collision term of

$$
C_{L}\left(f_{i}, f_{j}\right)=-\nabla_{v} \cdot \int_{\mathbb{R}^{3}} \mathrm{~d} v_{j} \quad \mathcal{Q}_{L}\left(\frac{1}{m_{j}} \frac{\partial}{\partial \mathbf{v}_{j}}-\frac{1}{m_{i}} \frac{\partial}{\partial \mathbf{v}_{i}}\right) f_{i} f_{j}
$$

where

$$
\mathcal{Q}_{L}=\frac{2 \pi}{m_{i}} \ln \Lambda_{i j}\left(\frac{q_{i} q_{j}}{4 \pi \epsilon_{0}}\right)^{2}\left(\frac{u^{2} \mathcal{I}-\mathbf{u u}}{u^{3}}\right)
$$


is the Landau collision operator, $\mathcal{I}$ is the identity matrix and $u=\left|\mathbf{v}_{i j}\right|$. It is equation (3), or its equivalent expression as the Vlasov-Fokker-Planck equation [49, 50], which is implicitly solved by small-angle collision algorithms.

We use a modified version of the TA [30] small-angle collision algorithm in our combined small- and large-angle algorithm. In the TA scheme, particles are paired together at every time step independent of $v_{i j}$ with the dependence on $v_{i j}$ only entering through the cumulative scattering angle between two particles. In the centre of mass frame, the scattering angles are picked based on the following statistical argument: since successive collisions are independent events, the central limit theorem ensures that for a large number of collisions, $N_{\text {coll }}$, the distribution in angle will be approximately Gaussian around the forward direction with a mean square angle

$$
\left\langle\Theta^{2}\right\rangle=N_{\text {coll }}\left\langle\theta^{2}\right\rangle
$$

Angular brackets signify an average value. $\left\langle\theta^{2}\right\rangle$ is most often calculated using the small-angle approximation so that

$$
\left\langle\theta^{2}\right\rangle=\frac{1}{\sigma} \int \theta^{2} \frac{\mathrm{d} \sigma}{\mathrm{d} \Omega} \mathrm{d} \Omega=\frac{\pi b_{\perp}^{2}}{2 \sigma} \int \frac{\theta^{2} \sin \theta}{\sin ^{4}(\theta / 2)} \mathrm{d} \theta \approx \frac{8 \pi b_{\perp}^{2}}{\sigma} \int \frac{\mathrm{d} \theta}{\theta}=8 \pi b_{\perp}^{2} \ln \Lambda / \sigma
$$

This integral is over $\theta \in(0, \pi)$. The number of collisions in a distance $\Delta s$ is given by $N_{\text {coll }}=n \sigma \Delta s=n \sigma v_{i j} \Delta t$ with $n=\min \left\{n_{i}, n_{j}\right\}$ and $\Delta t$ the timestep, so

$$
\left\langle\Theta^{2}\right\rangle=n \Delta t v_{i j} 8 \pi b_{\perp}^{2} \ln \Lambda
$$

where

$$
\ln \Lambda=\frac{1}{2} \ln \left(\frac{b_{\perp}^{2}+\lambda_{\mathrm{D}}^{2}}{b_{\perp}^{2}}\right)
$$

A number of terms have been omitted in (5) due to the small-angle approximation but their inclusion makes almost no difference to the value of $\left\langle\Theta^{2}\right\rangle$. Scattering angles for the particle pairs are then given by $\theta \sim \mathcal{N}\left(0,\left\langle\Theta^{2}\right\rangle\right)$.

There are many alternative formulations of $\ln \Lambda$, and the one derived in equation (6) is naïve in the sense that it does not include some effects known to be important in this regime. These include quantum corrections, $\lambda_{\mathrm{D}}$ becoming of similar size to the inter-ion separation distance, and the $\mathcal{O}(1 / \ln \Lambda)$ corrections to scattering at long distances (small-angles) due to dynamical screening [51, 52]. In order to account for these effects, we use Gericke, Murillo, and Schlanges' Coulomb logarithm No. 6 (GMS6) [37]. This $\ln \Lambda$ has been benchmarked against molecular dynamics simulations, and is valid for $N_{\mathrm{D}} \gtrsim 10$ [39], where $N_{\mathrm{D}}$ is the number of particles in 
a Debye sphere. In the simulations presented, this condition is satisfied and ionion correlations are negligible. To include the extra physics encoded by the GMS6 logarithm requires the substitution of two of the variables in equation $(6) ; \lambda_{\mathrm{D}}$ is replaced by $b_{\max }$, and $b_{\perp}$ with $b_{\min }$, where

$$
b_{\max }^{2} \equiv \lambda_{\mathrm{D}}^{2}+r_{0}^{2} ; \quad b_{\min }^{2} \equiv \lambda_{\mathrm{dB}}^{2}+b_{\perp}^{2}
$$

where the total ion sphere radius is $r_{0}=\left(4 \pi \sum_{i} n_{i} / 3\right)^{-1 / 3}$, and $\lambda_{\mathrm{dB}}$ is the de Broglie wavelength. This $\ln \Lambda$ and the usual TA algorithm form the basis for our extension to large-angles, and a code with these elements has been benchmarked in previous work [53]. Another small-angle algorithm may be used, as long as it is modified in the manner described in $\$ 3.2$.

\subsection{Small-angle and large-angle collision algorithm}

In order to extend the TA algorithm to include large-angle collisions it is necessary to split the range of scattering angles between the small- and large-angle algorithms, to reduce the strength of the small-angle algorithm appropriately, and to introduce our binary collision based large-angle algorithm. In \$2, the cut-off in impact parameter $b_{c}$ and its equivalent $\theta_{c}\left(b_{c}\right)$ are introduced. In the multiple-scattering-only TA algorithm, angles are picked from $\theta \sim \mathcal{N}\left(0,\left\langle\Theta^{2}\right\rangle\right)$ but the domain of integration in the derivation of the variance, $\left\langle\Theta^{2}\right\rangle$, must now be restricted to $\theta \in\left(0, \theta_{c}\right)$. Evaluating (4) for restricted $\theta$, the resulting variance is $\left\langle\Theta^{2}\right\rangle=n v_{i j} \Delta t 8 \pi b_{\perp}^{2} \ln \Lambda_{\mathrm{M}}$ where $\ln \Lambda_{\mathrm{M}}$ is a modified Coulomb logarithm which only includes small-angle collisions. It is given by

$$
\ln \Lambda_{\mathrm{M}}=\frac{1}{2} \ln \left(\frac{b_{\min }^{2}+b_{\max }^{2}}{b_{\min }^{2}+b_{c}^{2}}\right)
$$

Subscripts ' $M$ ' and ' $L$ ' henceforth refer to multiple and large-angle scattering respectively. This modification still allows for the use of the replacements in the GMS6 $\ln \Lambda$. Similarly, any theory restricted in angle and including extra corrections due to long-distance, small-angle effects could be used in place of equation (8).

In addition to the reduced strength multiple small-angle collisions, the large-angle collisions must be included in the algorithm. Rather than implicitly solving (3), it is equation (1) for deflections $\theta>\theta_{c}$ that should be solved. As described in $\$ 2$, large-angle collisions are instantaneous, with particles following the full Rutherford collision trajectory. There has been great success in modelling these types of collisions in Monte Carlo particle transport [54, 55], where, rather than explicitly solve a transport equation, the transport process is Markov-like with the probability of the next interaction depending only upon the current state of the particle. The same 
technique is deployed here to model the binary, large-angle Coulomb collisions within a plasma. Although we use this technique to provide a general method for simulating large-angle Coulomb collisions, there is an analytically tractable special case of their effects on a plasma which is used as a benchmark of the algorithm in 84.1 . The novelty of this approach to large-angle collisions relative to previous work on knockon ions is that other plasma evolution problems may be solved without, for instance, the assumption of a cold background, or that of restricting solutions to a single generation of knock-on ions.

The small-angle collisions proceed as in $\$ 3.1$, except with $\ln \Lambda_{\mathrm{M}}$ in place of $\ln \Lambda$. A separate large-angle algorithm is run in which particle pairs are selected locally and randomly using the TA particle pairing scheme. Given a pair of particles $i$ and $j$, and small $\Delta t$, the probability of a large-angle collision is given by

$$
P_{\mathrm{L}}=\sigma_{\mathrm{L}} n v_{i j} \Delta t
$$

The total cross-section for a large-angle collision is

$$
\sigma_{\mathrm{L}}=\frac{\pi b_{\perp}^{2}}{2} \int_{\theta_{c}}^{\pi} \frac{\sin \theta}{\sin ^{4}\left(\frac{\theta}{2}\right)} d \theta=\pi b_{c}^{2}
$$

from equation (2). Note that $P_{\mathrm{L}}=P_{\mathrm{L}}\left(v_{i j}\right)$, in contrast with the multiple-scattering methods based on the Vlasov-Fokker-Planck equation [33, in which particles are always collided at every time step but with a scattering angle dependent upon $v_{i j}$.

Large-angle collisions are carried out for a pair if $u \in \mathcal{U}(0,1)<P_{\mathrm{L}}$, otherwise no collision takes place. In the case that a large-angle collision occurs, a scattering angle in the centre of mass frame must be selected in accordance with equation (2). Defining

$$
\frac{\mathrm{d} \sigma}{\mathrm{d} \theta}=\frac{\pi b_{\perp}^{2}}{2} \frac{\sin \theta}{\sin ^{4}\left(\frac{\theta}{2}\right)}
$$

then the large-angle scattering probability density function given that a collision occurs is defined as

$$
\mathcal{P}(\theta) \mathrm{d} \theta=\frac{1}{\sigma} \frac{\mathrm{d} \sigma}{\mathrm{d} \theta} \mathrm{d} \theta
$$

This may be integrated to give $\mathcal{C}_{\mathrm{L}}(x)$, a cumulative density function in $\theta$ for largeangle Coulomb collisions;

$$
\mathcal{C}_{\mathrm{L}}(x)=\int_{\theta_{c}}^{x} \mathcal{P}(\theta) \mathrm{d} \theta=-\frac{b_{\perp}^{2}}{b_{c}^{2}}\left[\frac{1}{\sin ^{2}\left(\frac{\theta}{2}\right)}\right]_{\theta_{c}}^{x}
$$


In order to produce randomly generated values of $\theta$ distributed according to the Rutherford differential cross-section, it is then only necessary to substitute random numbers $u \sim \mathcal{U}(0,1)$ into the inverted cumulative distribution function to produce the centre of mass scattering angles;

$$
\theta=\mathcal{C}_{\mathrm{L}}^{-1}(u)=2 \sin ^{-1}\left[\left(\frac{b_{\perp}^{2}}{b_{\perp}^{2}+b_{c}^{2}(1-u)}\right)^{1 / 2}\right]
$$

Note that $\mathcal{C}_{\mathrm{L}}^{-1}(1)=\pi$ and $\mathcal{C}_{\mathrm{L}}^{-1}(0)=\theta_{c}$

In Monte Carlo particle transport with only 'large-angle' or 'hard' scattering events and no feedback between the particles, the distance to the next collision, $\Delta s$, may be sampled and particles moved to the point where they undergo a collision. In this case, there is a continuous energy loss due to collisions in addition to large-angle collisions and feedback between all particles, so large-angle collisions must occur with a certain probability per time step in line with the particles' properties changing due to the cumulative scattering mechanism. Hence the large-angle algorithm attempts to collide particles at every time step, but 'rejects' those pairings for which no collision takes place according to $P_{\mathrm{L}}$. With too large a time step, the probability of largeangle collisions will increase as $P_{\mathrm{L}} \propto \Delta t$ and $P_{\mathrm{L}}>1$ is possible. Choosing $\Delta t \ll$ $1 /\left(\min \left\{n_{i}, n_{j}\right\}\left\langle v_{i j} \pi b_{c}^{2}\right\rangle\right)$ ensures that this happens rarely and defines the time scale required to resolve the physics. However, as $P_{\mathrm{L}}=P_{\mathrm{L}}\left(v_{i j}\right)$, there may be times when most collisions are resolved, but occasionally there are interactions in which $v_{i j}$ causes $P_{\mathrm{L}}>1$. This is a natural consequence of the choice of $\Delta t$ reflecting an aggregate property of the plasma. In the small-angle only algorithms, this failure is typically dealt with by choosing $\theta \in \mathcal{U}(0, \pi)$ as the scattering angle. In the largeangle algorithm $P_{\mathrm{L}}>1$ is turned into an integer, $\left\lfloor P_{\mathrm{L}}\right\rfloor$, and a remainder $P_{\mathrm{D}}-\left\lfloor P_{\mathrm{D}}\right\rfloor$. $\left\lfloor P_{\mathrm{L}}\right\rfloor$ is the floor function, which returns the largest integer not greater than $P_{\mathrm{L}}$. The integer number of collisions is carried out, followed by another collision if $u \in$ $\mathcal{U}(0,1)<P_{\mathrm{D}}-\left\lfloor P_{\mathrm{D}}\right\rfloor . u$ is not re-used as at least one collision occurs if $P_{\mathrm{L}}>1$, and the random number comparison is with the non-integer portion of $P_{\mathrm{L}}$, ensuring that the number of large-angle collisions is insensitive to $\Delta t$. This preserves the $\mathcal{O}(N)$ operations scaling for $N$ computational particles as the number of collisions of both types is $\left(1+P_{\mathrm{L}}\right) N$, with $P_{\mathrm{L}}$ independent of $N$.

In order to show that the large-angle collision cross-section has the expected $\mathcal{O}(1 / \ln \Lambda)$ size relative to small-angle collisions in this theory, it is compared to the small-angle particle slowing cross-section as defined by the first kinetic cross-section,

$$
\sigma_{1, \mathrm{M}}=\int(1-\cos \theta) \mathrm{d} \sigma=4 \pi b_{\perp}^{2} \ln \Lambda_{\mathrm{M}}
$$




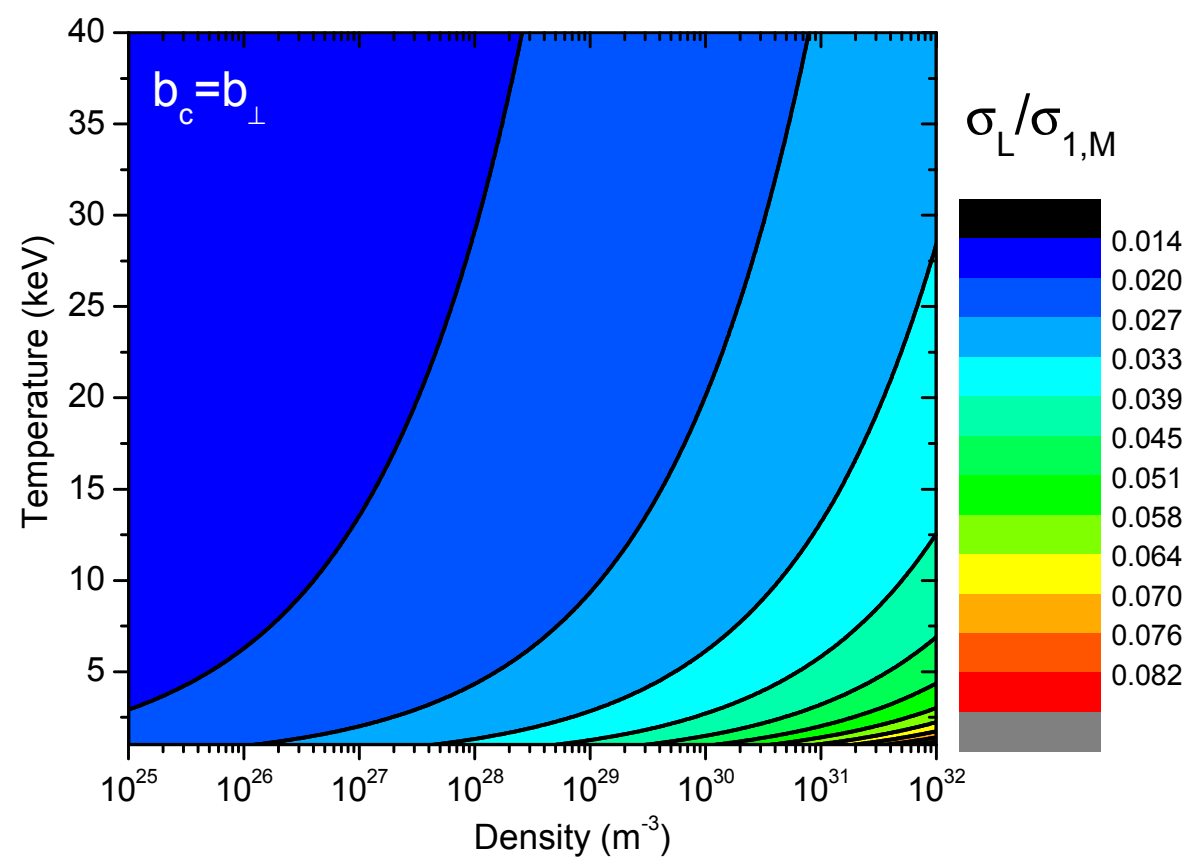

Figure 1: The ratio of the large-angle collision cross-section to the multiple small-angle first kinetic cross-sections for a deuterium-tritium Coulomb collision with $b_{c}=b_{\perp}$ in a deuterium-tritiumelectron plasma.

with a cut-off applied at $b=b_{c}$. For the particular case of $b_{c}=b_{\perp}$, the ratio of the large-angle collision cross-section $\sigma_{\mathrm{L}}=\pi b_{c}^{2}$ to $\sigma_{1, \mathrm{M}}$ is shown in Fig. 1 for deuteriumtritium collisions in a deuterium-tritium-electron plasma and is $1 /\left(4 \ln \Lambda_{\mathrm{M}}\right) \approx 1 /(4 \ln \Lambda)$ and so tends to zero for large $\ln \Lambda$.

\section{Benchmarking the algorithm}

\subsection{Rate of generation of knock-on ions}

A benchmark of the method is provided by comparison with Ryutov's theory, developed by Ryutov [10] and Helander [44], for the source rate of knock-on ions. This theory has been successfully used for predictions [15, 16] of the neutron spectra from magnetic confinement fusion experiments, and found to be in agreement with experimental data [18]. Given a fast particle $\alpha$, the source rate of knock-ons of ions of species $i$ per unit volume per unit time is given by

$$
Q_{i}\left(E_{i}^{\prime}\right) \mathrm{d} E_{i}^{\prime}=Q\left(v_{i}^{\prime}\right) 4 \pi v_{i}^{\prime 2} \mathrm{~d} v_{i}^{\prime}=8 \pi \gamma^{2} n_{i} 4 \pi v_{i}^{\prime} \mathrm{d} v_{i}^{\prime} \int_{\gamma v_{i}^{\prime}}^{\infty}\left(\frac{\mathrm{d} \sigma}{\mathrm{d} \Omega}\right) f_{\alpha}\left(v_{\alpha}\right) v_{\alpha} \mathrm{d} v_{\alpha}
$$


under the assumption that $i$ is cold. $\gamma=m_{i} /\left(2 m_{i \alpha}\right)$ and $\left(\frac{\mathrm{d} \sigma}{\mathrm{d} \Omega}\right)=\left(\frac{q_{i} q_{\alpha}}{4 \pi \epsilon_{0}}\right)^{2}\left(\frac{1}{\gamma m_{i} v_{i}^{\prime 2}}\right)^{2}$ is the Rutherford cross-section. Note that $v_{i}^{\prime}=\frac{v_{\alpha}}{\gamma} \sin \left(\frac{\theta}{2}\right)$. In the simple case when there is a mono-energetic distribution of $\alpha$ particles with speed $v_{\alpha}^{*}$,

$$
f_{\alpha}\left(v_{\alpha}\right)=n_{\alpha} \delta\left(v_{\alpha}-v_{\alpha}^{*}\right) / 4 \pi v_{\alpha}^{2}
$$

then

$$
Q_{i}\left(E_{i}^{\prime}\right) \mathrm{d} E_{i}^{\prime}=\left(\frac{q_{i} q_{\alpha}}{4 \pi \epsilon_{0}}\right)^{2} \frac{2 \pi n_{i} n_{\alpha}}{v_{\alpha}^{*} m_{i} E_{i}^{\prime 2}} \mathrm{~d} E_{i}^{\prime}
$$

On the assumption that $b_{c}=b_{\perp}$, the maximum energy transfer is $E_{i, \max }^{\prime}=4 E_{\alpha}^{*} m_{i \alpha}^{2} /\left(m_{i} m_{\alpha}\right)$ (determined by $b=0$ ), and the minimum is $E_{i, \min }^{\prime}=E_{i, \max }^{\prime} / 2$ (determined by $\left.b_{c}=b_{\perp}\right)$, so that the total rate of knock-ons is

$$
Q_{i}^{\text {tot }}=\left(\frac{q_{i} q_{\alpha}}{4 \pi \epsilon_{0}}\right)^{2} \frac{\pi n_{i} n_{\alpha}}{\left(v_{\alpha}^{*}\right)^{3} m_{i \alpha}^{2}}
$$

Fig. 2 shows the rate of generation of knock-on deuterium ions according to the theory in equation (11) against the results of our Monte Carlo simulation with large-angle collisions. Both methods assume $b_{c}=b_{\perp}$ and initial conditions of $n_{\alpha}=$ $n_{\mathrm{D}}=5 \times 10^{31} \mathrm{~m}^{-3}$, with an isotropic, mono-energetic $\alpha$ particle distribution with $E_{\alpha}^{*}=3.54 \mathrm{MeV}$, and cold deuterium. The Monte Carlo simulation with large-angle collisions is restricted to only producing the first generation of knock-ons, in order to compare against the Ryutov theory, which makes the same assumption. The simple scenario of two mono-energetic distribution functions allows for a direct comparison against an analytical expression. The initial energy of the $\alpha$ particles is indicated on Fig. 2. The sharp upper cut-off in the final energy of the knock-ons is caused by the kinematics and that the initial $\alpha$ particle energy is set at a single value. The sharp lower cut-off is a consequence of using two mono-energetic distributions for the two colliding species, so that the value of $b_{\perp}$ is very similar for each collision. The value of $b_{\perp}$ then determines the maximum allowed final energy of the knock-on. In a more physical situation, in which the distributions are not mono-energetic, a range of initial energies of the colliding species cause a smoother lower cut-off in energy of the generated knock-on ions. The simulation only includes the interaction between $\alpha$ particles and deuterons.

In the Monte Carlo simulation shown in Fig. 2, the rate of generation of knockons is calculated from $Q_{i}\left(E_{i}^{\prime}\right)=\mathrm{N}\left(E_{i}^{\prime}, E_{i}^{\prime}+\mathrm{d} E_{i}^{\prime}\right) w / t$ where $t$ is the time passed in the simulation, $\mathrm{N}\left(E_{i}^{\prime}, E_{i}^{\prime}+\mathrm{d} E_{i}^{\prime}\right)$ counts the number of knock-on ions of species $i$ with energies between $E_{i}^{\prime}$ and $E_{i}^{\prime}+\mathrm{d} E_{i}^{\prime}$, and $w$ is the weighting of particles, defined as $w=\sum_{j} n_{j} / \sum_{j} N_{j}$ with $N_{j}$ the total number of simulation particles of type $j$. The agreement between the Monte Carlo simulation and the theory is excellent. 


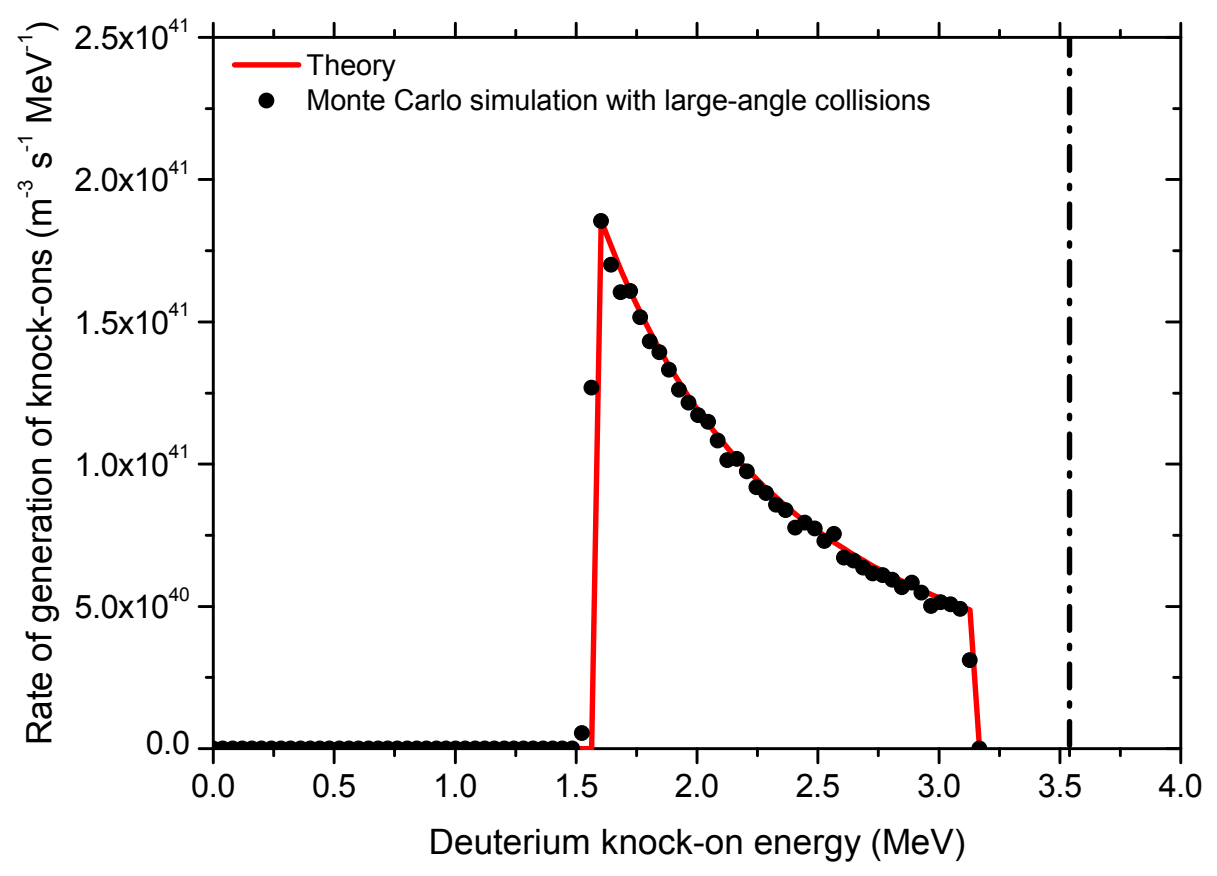

Figure 2: Benchmarking the source rate, $Q_{\mathrm{D}}$, of knock-on deuterium ions due to fast $\alpha$ particles incident on cold deuterium assuming that the cut-off takes the value $b_{c}=b_{\perp}$. The theory is described by equation (11). The dashed-dotted vertical line indicates the initial alpha particle energy.

\subsection{Temperature equilibration}

In addition to creating knock-on ions at the relevant energy and frequency, the combined small- and large-angle model should reproduce known results on temperature equilibration in circumstances where the distribution functions of the two equilibrating ion species remain well-described by a Maxwell-Boltzmann distribution. Such a scenario is represented by the equilibration of deuterium and tritium at solid density with initial temperatures of 2 and $2.5 \mathrm{keV}$ respectively. Fig. 3 shows the equilibration of these two species over time with $b_{c}=0, b_{\perp}, 3 b_{\perp}$ and the rate of equilibration based on the GMS6 Coulomb logarithm [37]. Also included in all models are the collisions between electrons and electrons, and electrons and ions, all of which occur via small-angle collisions only. The electrons have an initial temperature of $2.5 \mathrm{keV}$.

Note that, in this scenario, it is unlikely that distortions would occur to ion distribution functions, as the average energies of the two species are close. This is confirmed by the excess kurtosis, defined by $\kappa=\int(E-\mu)^{4} f(E) \mathrm{d} E-3$ with 




Figure 3: The equilibration of two ion species over time with, and without, large-angle collisions in a regime in which they are expected to be unimportant. Also shown is the theoretical rate of equilibration.

$\mu$ the mean, which provides a metric for the deviation from a Maxwell-Boltzmann distribution, and satisfies $|\kappa|<0.1$ for all simulations considered, demonstrating that the addition of the large-angle Coulomb collisions make no difference to distribution functions, or equilibration, when the energy transfers are small.

\section{Application to generation of knock-ons in an ICF hotspot}

We apply the model to a simplified inertial confinement fusion hotspot [56] in conditions which favour the generation of knock-on ions in order to demonstrate the distortions to ion distribution functions which can result. The hotspot contains deuterium, tritium, and electrons (in charge balance) initiated in thermal equilibrium at $T=3 \mathrm{keV}$ and $n_{\mathrm{D}}=n_{\mathrm{T}}=4.52 \times 10^{31} \mathrm{~m}^{-3} .5 \%$ alpha particles are added at the deuterium-tritium fusion production energy of $E=3.54 \mathrm{MeV}$. Fusion of deuterium and tritium is enabled, but the less likely deuterium-deuterium and tritium-tritium fusion reactions are omitted. The fusion routine has been benchmarked against other work [57]. Hotspot energy loss mechanisms, such as thermal conduction, radiation, 


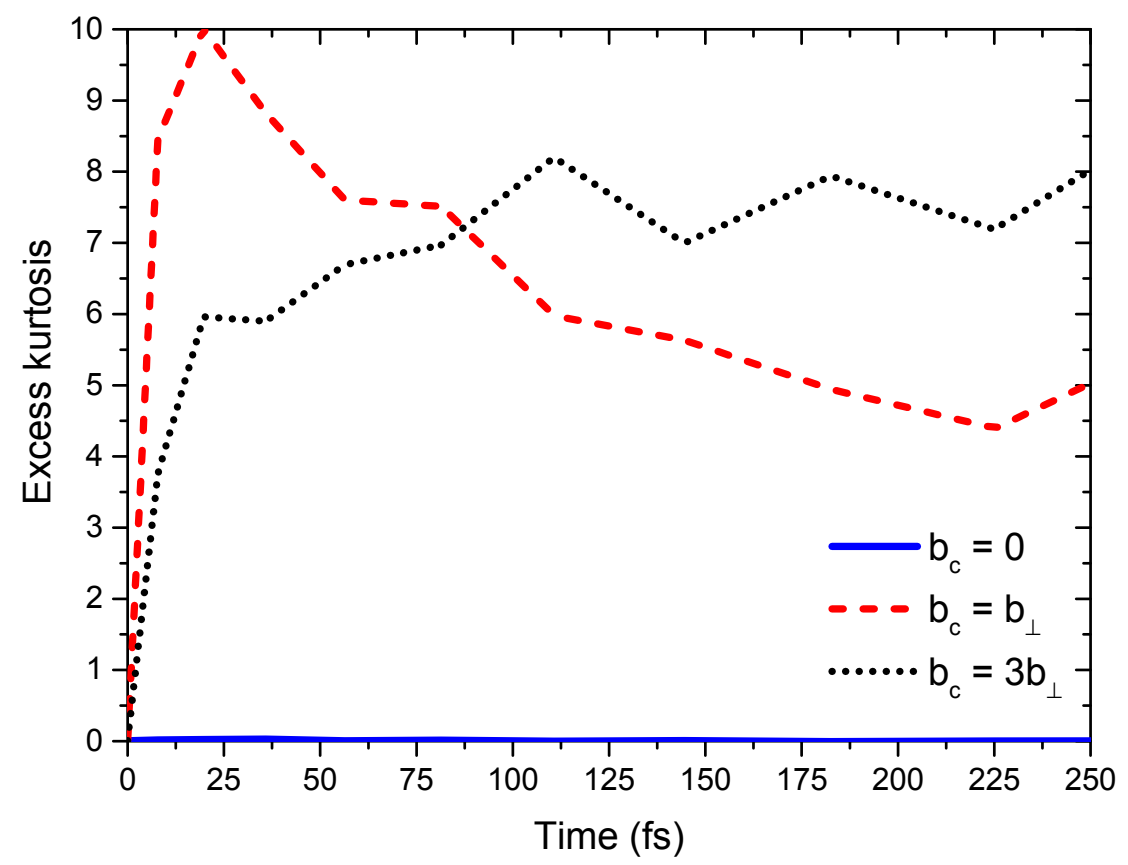

Figure 4: Deuterium excess kurtosis, $\kappa$, over time; this demonstrates the departure from thermal equilibrium caused by fast $\alpha$-particles 'knocking-on' background deuterons to many times their initial energy.

and $\alpha$ escape, are not included. As described in \$1, large-angle collisions are only modelled between ion species; a summary of the included interactions is given in Table 1.

Three models for $b_{c}$ are simulated, and the excess kurtosis of the deuterium ions for these simulations are plotted as a function of time as shown in Fig. 4. As no loss mechanisms are included in these hotspot simulations, the increases in yield feed back into higher temperatures, and further fusion reactions. The timescales examined, of a few hundred fs, are short relative to the burn time of an ICF capsule of 20-50 ps.

\begin{tabular}{l|cl} 
Species & Electron & Ion \\
\hline Electron & Small-angle, $b_{c}=0$ & Small-angle, $b_{c}=0$ \\
Ion & Small-angle, $b_{c}=0$ & $\begin{array}{l}\text { Large- and small-angle, } b_{c} \\
\text { as labelled }\end{array}$
\end{tabular}

Table 1: Summary of the enabled interactions for the hotspot simulations 
As $\kappa=0$ in thermal equilibrium, the two simulations using $b_{c} \neq 0$ in Fig. 4 show that the deuterium distribution function has been distorted by the slowing of the $\alpha$-particles when large-angle Coulomb collisions are accounted for. In the case without large-angle Coulomb collisions, the energy loss of the $\alpha$ s is more evenly spread over the background ions. Because faster particles have an energy deposition time $\tau \propto 1 / v^{3}$, depositing the $\alpha$ energy in fewer, more energetic deuterium and tritium ions when including large-angle collisions means that the ion distribution functions take longer to equilibrate than when that energy is deposited more evenly.

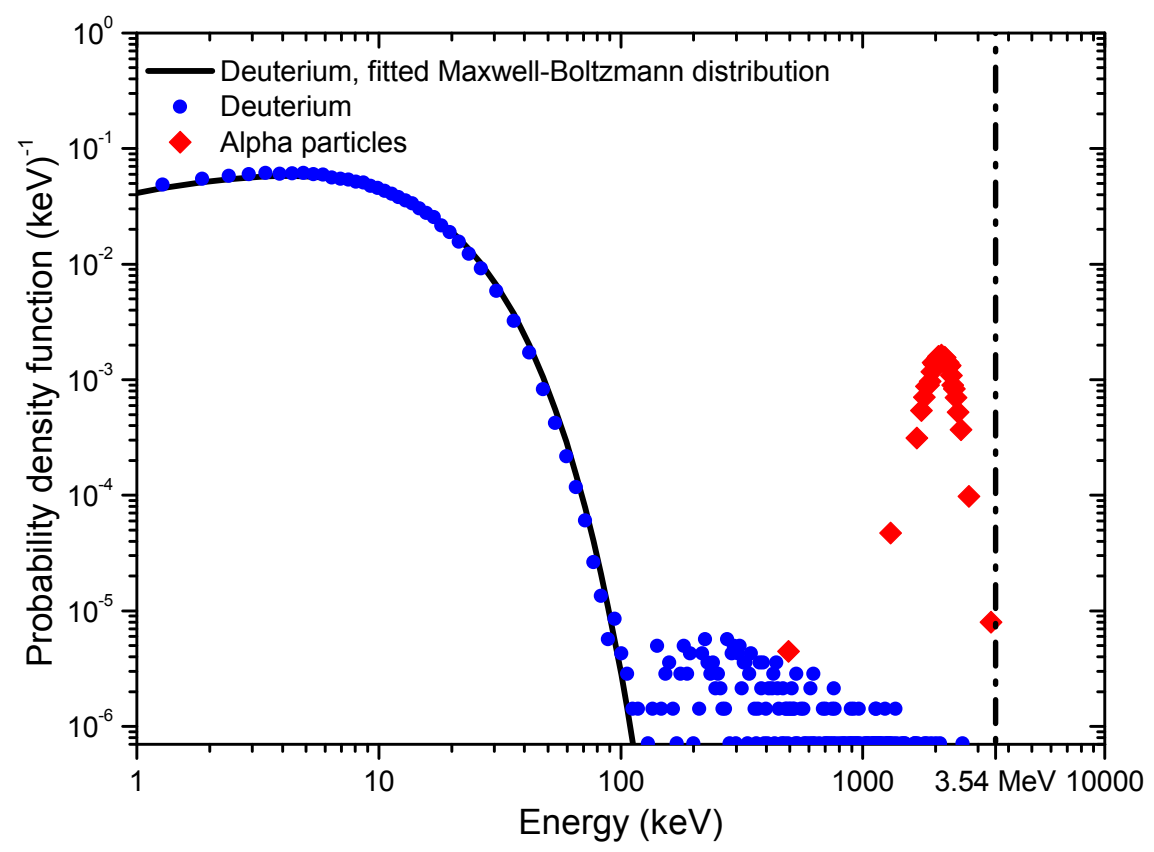

Figure 5: Deuterium and $\alpha$ distributions from the Monte Carlo simulation for $b_{c}=3 b_{\perp}$ after $t=590 \mathrm{fs}$. Also shown is a Maxwell-Boltzmann distribution fitted with a temperature $T_{\mathrm{D}}=$ $2\left\langle E_{\mathrm{D}}\right\rangle / 3=8 \mathrm{keV}$. The probability density functions are normalised to unity.

A snapshot of deuterium and $\alpha$ distribution functions is provided in Fig. 5. The fitted Maxwell-Boltzmann distribution for deuterium, also shown, has a temperature such that $3 T_{\mathrm{D}} / 2=\left\langle E_{\mathrm{D}}\right\rangle$ and provides a good fit for the bulk of the distribution function. However, it underestimates the low energy part of the distribution relative to the simulated distribution function, and, importantly, overestimates the region which contributes most to the deuterium-tritum fusion reactivity. A high energy tail is clearly present due to the knock-on effect, and its probability density is orders of magnitude higher than the tail of the Maxwell-Boltzmann distribution. The small- 
angle-only, $b_{c}=0$ deuterium and tritium distribution functions remain Maxwellian, while the $b_{c}=b_{\perp}$ distribution shows less distortion than $b_{c}=3 b_{\perp}$ and more than $b_{c}=0$ as expected.

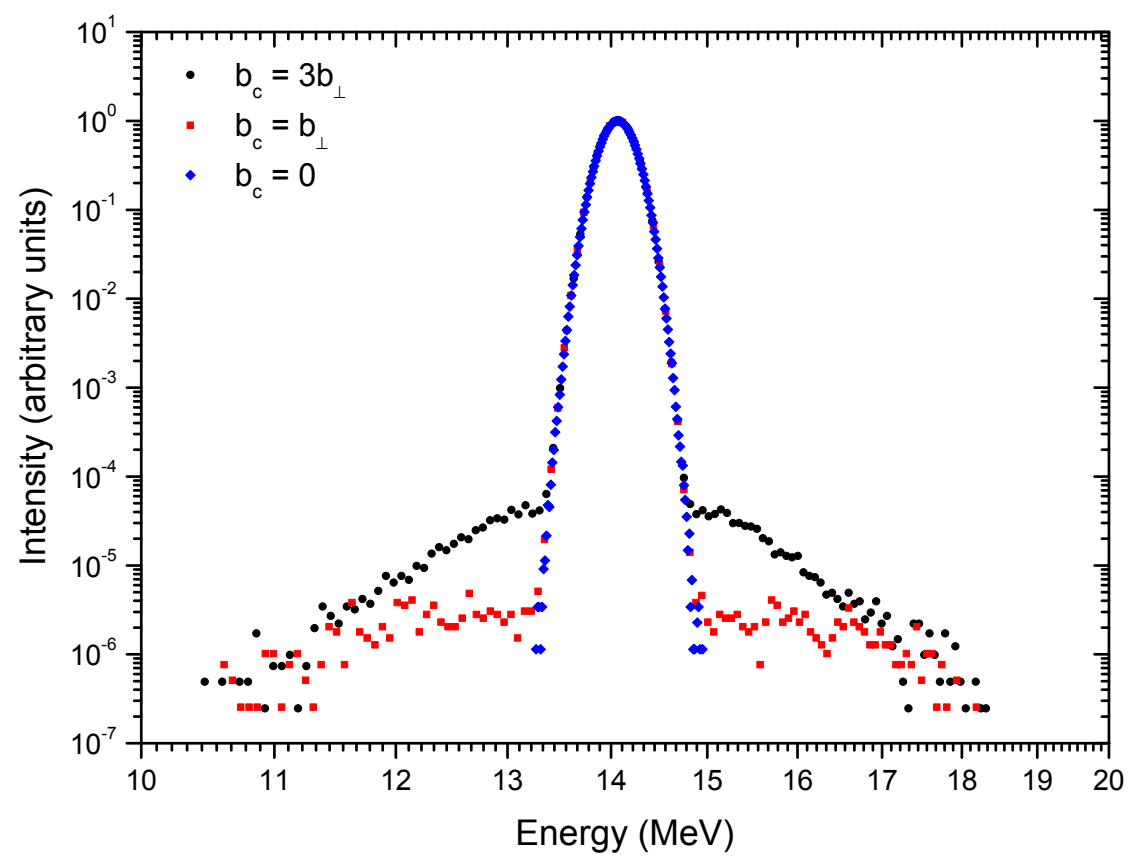

Figure 6: The time-integrated neutron spectrum at $t=150$ fs after the start of the simulation, when the ion temperature has reached $4 \mathrm{keV}$.

The strong dependence of the fusion cross-section on collision energy ensures that even small distortions to the high energy portion of the fusing ions' distribution functions can have consequences for the neutron spectrum. These effects are shown in Fig. 6, which is the time-integrated neutron spectrum at $t=150 \mathrm{fs}$. Though the primary neutron spectra, from thermal deuterium and tritium fusion, are in agreement, the spectra due to knock-on ions fusing are dependent on the value of $b_{c}$. The fusion of knock-on ions with background ions cause the tails to form in the neutron spectra. The tails have an intensity which is orders of magnitude lower than the primary neutron spectrum.

In this scenario, the change to distribution functions does result in a change to yield over the time scales of 100s of fs considered. For initial ion temperatures above $1 \mathrm{keV}$, we find that the effect of the inclusion of large-angle Coulomb collisions is to take probability density from the peak of the fusion cross-section and re-distribute 


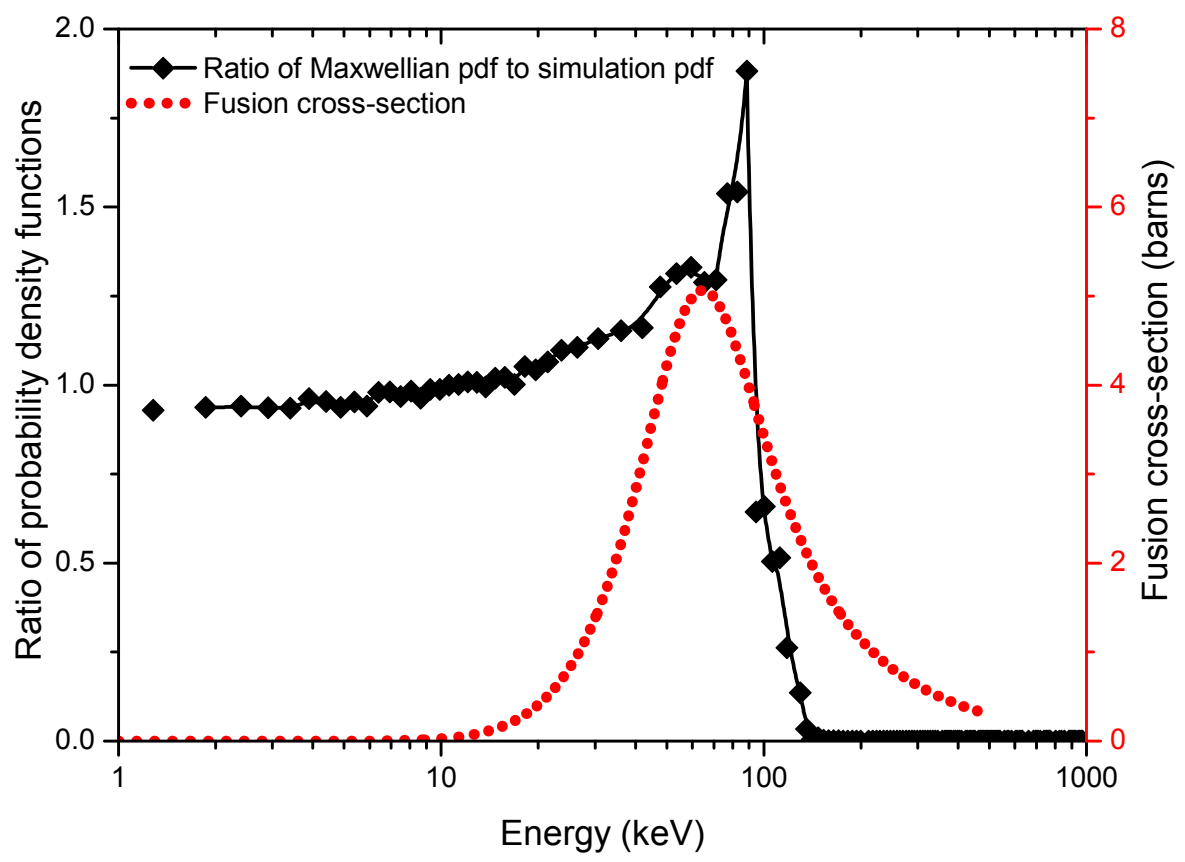

Figure 7: The ratio of a Maxwell-Boltzmann distribution to the simulated probability density function of deuterium at $t=590 \mathrm{fs}$ for $b_{c}=3 b_{\perp}$. Also shown is the fusion cross-section. The simulated distribution function is depleted at the peak of the cross-section, with the probability density shifted to higher energies.

it above the peak, so as to reduce the reactivity and therefore reduce the yield. This can be seen clearly in Fig. 5, and is made explicit in Fig. 7 which shows that the simulated deuterium distribution function is depleted relative to a fitted MaxwellBoltzmann distribution around the peak of the fusion cross-section, but has more probability density as the cross-section decreases at energies above the peak. Despite the large difference at the peak of the fusion cross-section, the reactivity is dominated by the bulk distribution and the effects on yield relative to the small-angle only case are small, giving a less than $8 \%$ reduction for the values of $b_{c}$ considered.

It should be noted that experimental neutron spectra are the product of other effects in addition to Coulomb collision charged-particle induced knock-ons; these include the down-scattering of produced neutrons and neutron-induced knock-ons, nuclear elastic scattering [16, 58, inelastic scattering processes and light nuclei breakup reactions [20], capsule anisotropies and consideration of the scattering in the cold fuel and ablator, and detector sensitivity. There is a possibility that the correct model for $b_{c}$ could be determined from data obtained from ICF experiments, but this would 
require neutron spectra accurate over many orders of magnitude and self-consistent inclusion of these other processes.

\section{Conclusion}

We have provided a new technique to evaluate the effects of large-angle Coulomb collisions in plasmas with $\ln \Lambda \gtrsim 2$, and shown that their inclusion has a demonstrable effect on the tails of ion distribution functions when a source of fast particles is present. This technique allows for the self-consistent evolution of ion distribution functions under the influence of large-angle Coulomb collisions, and includes knockon ions of all generations. In the context of ICF, the strong dependence of the fusion reactivity on the tails of the ion distribution functions means that the neutron spectrum is significantly changed by the inclusion of large-angle Coulomb collisions. The effects included by our method cannot be predicted by fluid or Vlasov-FokkerPlanck models, which are incapable of reproducing the large jumps in particle energy which occur due to large-angle collisions, and the generation of ion knock-ons is treated to all orders with self-consistent evolution of species' distribution functions.

\section{Acknowledgments}

A. E. T. was supported by an EPSRC Doctoral Prize Fellowship while undertaking this research. The authors would like to thank Warren Garbett for help-

ful discussions. This work was funded under EPSRC grants EP/L504786/1 and $\mathrm{EP} / \mathrm{K} 028464 / 1$.

\section{References}

[1] B. A. Trubnikov, Reviews of Plasma Physics, Vol. 1, Consultants Bureau, 1965.

[2] C. P. Ridgers, C. S. Brady, R. Duclous, J. G. Kirk, K. Bennett, T. D. Arber, A. P. L. Robinson, A. R. Bell, Dense electron-positron plasmas and ultraintense $\gamma$ rays from laser-irradiated solids, Phys. Rev. Lett. 108 (2012) 165006. doi: 10.1103/PhysRevLett.108.165006.

URL http://link .aps .org/doi/10.1103/PhysRevLett.108.165006

[3] S. Son, N. Fisch, Ignition regime for fusion in a degenerate plasma, Physics Letters A 356 (1) (2006) 72 - 78. doi:http://dx.doi.org/10.1016/j. physleta.2006.03.065.

URL http://www.sciencedirect.com/science/article/pii/ S0375960106005068 
[4] J. P. Cox, R. T. Giuli, Principles of stellar structure volume II: applications to stars, Gordon and Breach Science Publishers, 1968.

[5] S. J. Rose, The effect of degeneracy on the scattering contribution to the radiative opacity, The Astrophysical Journal Letters 453 (1) (1995) L45.

URL http://stacks .iop.org/1538-4357/453/i=1/a=L45

[6] J. D. Lindl, Development of the indirect-drive approach to inertial confinement fusion and the target physics basis for ignition and gain, Physics of Plasmas 2 (11) (1995) 3933-4024. doi:10.1063/1.871025.

URL http://link .aip.org/link/?PHP/2/3933/1

[7] J. Lindl, O. Landen, J. Edwards, E. Moses, N. Team, Review of the national ignition campaign 2009-2012, Physics of Plasmas 21 (2) (2014) -. doi:http://dx.doi.org/10.1063/1.4865400.

URL http://scitation.aip.org/content/aip/journal/pop/21/2/10. $1063 / 1.4865400$

[8] M. J. Edwards, J. D. Lindl, B. K. Spears, S. V. Weber, L. J. Atherton, D. L. Bleuel, D. K. Bradley, D. A. Callahan, C. J. Cerjan, D. Clark, G. W. Collins, J. E. Fair, R. J. Fortner, S. H. Glenzer, S. W. Haan, B. A. Hammel, A. V. Hamza, S. P. Hatchett, N. Izumi, B. Jacoby, O. S. Jones, J. A. Koch, B. J. Kozioziemski, O. L. Landen, R. Lerche, B. J. MacGowan, A. J. MacKinnon, E. R. Mapoles, M. M. Marinak, M. Moran, E. I. Moses, D. H. Munro, D. H. Schneider, S. M. Sepke, D. A. Shaughnessy, P. T. Springer, R. Tommasini, L. Bernstein, W. Stoeffl, R. Betti, T. R. Boehly, T. C. Sangster, V. Y. Glebov, P. W. McKenty, S. P. Regan, D. H. Edgell, J. P. Knauer, C. Stoeckl, D. R. Harding, S. Batha, G. Grim, H. W. Herrmann, G. Kyrala, M. Wilke, D. C. Wilson, J. Frenje, R. Petrasso, K. Moreno, H. Huang, K. C. Chen, E. Giraldez, J. D. Kilkenny, M. Mauldin, N. Hein, M. Hoppe, A. Nikroo, R. J. Leeper, The experimental plan for cryogenic layered target implosions on the national ignition facility - the inertial confinement approach to fusion, Physics of Plasmas 18 (5) (2011) 051003. doi : 10.1063/1.3592173. URL http://link .aip.org/link/?PHP/18/051003/1

[9] H. Matsuura, Y. Nakao, Effect of nuclear elastic scattering on ion heating characteristics in deuterium-tritium thermonuclear plasmas, Physics of Plasmas 13 (6) (2006) 062507. doi:10.1063/1.2206550.

URL http://link.aip.org/link/?PHP/13/062507/1 
[10] D. Ryutov, Energetic ion population formed in close collision with fusion alphaparticles, Physica Scripta 45 (2) (1992) 153.

URL http: //stacks . iop.org/1402-4896/45/i=2/a=014

[11] M. Sherlock, S. J. Rose, The persistence of Maxwellian d and t distributions during burn in inertial confinement fusion, High Energy Density Physics 5 (1-2) (2009) 27 - 30. doi:10.1016/j.hedp.2008.11.001.

URL http://wwW.sciencedirect.com/science/article/pii/ S1574181808000372

[12] D. Michta, F. Graziani, T. Luu, J. Pruet, Effects of nonequilibrium particle distributions in deuterium-tritium burning, Physics of Plasmas 17 (1) (2010) 012707. doi:10.1063/1.3276103. URL http://link .aip.org/link/?PHP/17/012707/1

[13] B. J. Albright, K. Molvig, C.-K. Huang, A. N. Simakov, E. S. Dodd, N. M. Hoffman, G. Kagan, P. F. Schmit, Revised knudsen-layer reduction of fusion reactivity, Physics of Plasmas 20 (12) (2013) -. doi:http://dx.doi.org/10.1063/1.4833639.

URL http://scitation.aip.org/content/aip/journal/pop/20/12/10. $1063 / 1.4833639$

[14] K. Molvig, N. M. Hoffman, B. J. Albright, E. M. Nelson, R. B. Webster, Knudsen layer reduction of fusion reactivity, Phys. Rev. Lett. 109 (2012) 095001. doi: 10.1103/PhysRevLett.109.095001.

URL http://link .aps .org/doi/10.1103/PhysRevLett.109.095001

[15] G. Gorini, L. Ballabio, J. Källne, Alpha-particle kinetic effects in the neutron emission of burning dt plasmas, Review of Scientific Instruments 66 (1) (1995) 936-938. doi:10.1063/1.1146213.

URL http://link .aip.org/link/?RSI/66/936/1

[16] L. Ballabio, G. Gorini, J. Källne, $\alpha$-particle knock-on signature in the neutron emission of dt plasmas, Phys. Rev. E 55 (1997) 3358-3368. doi:10.1103/ PhysRevE.55.3358.

URL http://link .aps .org/doi/10.1103/PhysRevE.55.3358

[17] A. Andrade, G. M. Hale, Nuclear effects on ion heating within the small-angle charged-particle elastic-scattering regime, Phys. Rev. A 30 (1984) 1940-1947. doi:10.1103/PhysRevA.30.1940.

URL http://link.aps .org/doi/10.1103/PhysRevA .30.1940 
[18] J. Källne, L. Ballabio, J. Frenje, S. Conroy, G. Ericsson, M. Tardocchi, E. Traneus, G. Gorini, Observation of the alpha particle "knock-on" neutron emission from magnetically confined dt fusion plasmas, Phys Rev Lett 85 (6) (2000) 1246-9.

[19] R. K. Fisher, P. B. Parks, J. M. McChesney, M. N. Rosenbluth, Fast alpha particle diagnostics using knock-on ion tails, Nuclear Fusion 34 (10) (1994) 1291.

URL http: //stacks . iop .org/0029-5515/34/i=10/a=I01

[20] J. A. Frenje, R. Bionta, E. J. Bond, J. A. Caggiano, D. T. Casey, C. Cerjan, J. Edwards, M. Eckart, D. N. Fittinghoff, S. Friedrich, V. Y. Glebov, S. Glenzer, G. Grim, S. Haan, R. Hatarik, S. Hatchett, M. G. Johnson, O. S. Jones, J. D. Kilkenny, J. P. Knauer, O. Landen, R. Leeper, S. L. Pape, R. Lerche, C. K. Li, A. Mackinnon, J. McNaney, F. E. Merrill, M. Moran, D. H. Munro, T. J. Murphy, R. D. Petrasso, R. Rygg, T. C. Sangster, F. H. Séguin, S. Sepke, B. Spears, P. Springer, C. Stoeckl, D. C. Wilson, Diagnosing implosion performance at the National Ignition Facility (NIF) by means of neutron spectrometry, Nuclear Fusion 53 (4) (2013) 043014.

URL http://stacks . iop.org/0029-5515/53/i=4/a=043014

[21] F. Evans, Energy deposition of a fast deuteron in a hot deuterium plasma, Physics of Fluids 16 (7) (1973) 1011-1020. doi:10.1063/1.1694463.

URL http://link . aip.org/link/?PFL/16/1011/1

[22] C.-K. Li, R. D. Petrasso, Fokker-Planck equation for moderately coupled plasmas, Phys. Rev. Lett. 70 (1993) 3063-3066. doi:10.1103/PhysRevLett.70. 3063 .

URL http://link .aps .org/doi/10.1103/PhysRevLett.70.3063

[23] X. Zha, S. Han, Z. Xu, Y. Wang, Collision operator including large-angle scattering for moderately coupled plasma, High Energy Density Physics 2 (3-4) (2006) $70-76$. doi:10.1016/j.hedp.2006.05.001.

URL http://wWW.sciencedirect.com/science/article/pii/ S1574181806000085

[24] K. A. Brueckner, H. Brysk, R. S. Janda, Athermal neutrons and ions in a fusion plasma, Journal of Plasma Physics 11 (1974) 403-409. doi:10.1017/ S0022377800024764.

URL http://dx.doi .org/10.1017/S0022377800024764 
[25] Y. Afek, A. Dar, A. Peres, A. Ron, R. Shachar, D. Shvarts, The fusion of suprathermal ions in a dense plasma, Journal of Physics D: Applied Physics 11 (16) (1978) 2171.

URL http://stacks . iop.org/0022-3727/11/i=16/a=004

[26] A. A. Korotkov, A. Gondhalekar, R. J. Akers, Observation of MeV energy deuterons produced by knock-on collisions between deuterium-tritium fusion alpha-particles and plasma fuel ions, Physics of Plasmas 7 (3) (2000) 957-962. doi:10.1063/1.873894.

URL http://link . aip.org/link/?PHP/7/957/1

[27] R. S. Cohen, L. Spitzer, Jr., P. McRoutly, The electrical conductivity of an ionized gas, Phys. Rev. 80 (1950) 230-238. doi:10.1103/PhysRev.80.230.

URL http://link.aps.org/doi/10.1103/PhysRev.80.230

[28] D. V. Sivukhin, Reviews of Plasma Physics, Vol. 4, Consultants Bureau, 1966.

[29] E. C. Shoub, Failure of the Fokker-Planck approximation to the Boltzmann integral for (1/r) potentials, Physics of Fluids 30 (5) (1987) 1340. doi:10.1063/1.866508.

URL http://scitation.aip.org/content/aip/journal/pof1/30/5/10. $1063 / 1.866508$

[30] T. Takizuka, H. Abe, A binary collision model for plasma simulation with a particle code, Journal of Computational Physics 25 (1977) 205-219.

[31] K. Nanbu, Theory of cumulative small-angle collisions in plasmas, Physical Review E 55 (4) (1997) 4642-4652. doi:10.1103/PhysRevE.55.4642. URL http://link .aps .org/doi/10.1103/PhysRevE.55.4642

[32] M. Sherlock, A Monte-Carlo method for coulomb collisions in hybrid plasma models, Journal of Computational Physics 227 (4) (2008) 2286-2292. doi: $10.1016 / \mathrm{j} . \mathrm{jcp} .2007 .11 .037$.

URL http://linkinghub .elsevier . com/retrieve/pii/S0021999107005359

[33] A. V. Bobylev, I. F. Potapenko, Monte Carlo methods and their analysis for Coulomb collisions in multicomponent plasmas, Journal of Computational Physics 246 (2013) 123-144. doi:10.1016/j.jcp.2013.03.024.

URL http://dx.doi .org/10.1016/j . jcp.2013.03.024 
[34] E. Greenspan, D. Shvarts, A multigroup model for the slowing-down of energetic ions in plasmas, Nuclear Fusion 16 (2) (1976) 295.

URL http: //stacks . iop.org/0029-5515/16/i=2/a=012

[35] S. D. Baalrud, Transport coefficients in strongly coupled plasmas, Physics of Plasmas 19 (3) (2012) -. doi:http://dx.doi.org/10.1063/1.3690093.

URL http://scitation.aip.org/content/aip/journal/pop/19/3/10. $1063 / 1.3690093$

[36] L. S. Brown, D. L. Preston, Robert L. Singleton Jr., Charged particle motion in a highly ionized plasma, Physics Reports 410 (4) (2005) 237 - 333. doi:http://dx.doi.org/10.1016/j.physrep.2005.01.001. URL http://www.sciencedirect.com/science/article/pii/ S0370157305000037

[37] D. O. Gericke, M. S. Murillo, M. Schlanges, Dense plasma temperature equilibration in the binary collision approximation, Phys. Rev. E 65 (2002) 036418. doi:10.1103/PhysRevE.65.036418. URL http://link.aps.org/doi/10.1103/PhysRevE.65.036418

[38] G. Dimonte, J. Daligault, Molecular-dynamics simulations of electron-ion temperature relaxation in a classical coulomb plasma, Phys. Rev. Lett. 101 (2008) 135001. doi:10.1103/PhysRevLett.101.135001. URL http://link .aps .org/doi/10.1103/PhysRevLett.101.135001

[39] J. N. Glosli, F. R. Graziani, R. M. More, M. S. Murillo, F. H. Streitz, M. P. Surh, L. X. Benedict, S. Hau-Riege, A. B. Langdon, R. A. London, Molecular dynamics simulations of temperature equilibration in dense hydrogen, Phys. Rev. E 78 (2008) 025401. doi:10.1103/PhysRevE.78.025401. URL http://link.aps.org/doi/10.1103/PhysRevE.78.025401

[40] J. Vorberger, D. Gericke, Comparison of electron-ion energy transfer in dense plasmas obtained from numerical simulations and quantum kinetic theory, High Energy Density Physics 10 (2014) 1-8. doi:10.1016/j.hedp.2013.10.006. URL http://linkinghub.elsevier .com/retrieve/pii/S1574181813001778

[41] L. X. Benedict, M. P. Surh, J. I. Castor, S. A. Khairallah, H. D. Whitley, D. F. Richards, J. N. Glosli, M. S. Murillo, C. R. Scullard, P. E. Grabowski, D. Michta, F. R. Graziani, Molecular dynamics simulations and generalized lenard-balescu calculations of electron-ion temperature equilibration in plasmas, Phys. Rev. E 
86 (2012) 046406. doi:10.1103/PhysRevE.86.046406.

URL http://link.aps.org/doi/10.1103/PhysRevE.86.046406

[42] J. Hansen, I. McDonald, Thermal relaxation in a strongly coupled two-temperature plasma, Physics Letters A 97 (1-2) (1983) 42 - 44. doi:http://dx.doi.org/10.1016/0375-9601(83)90097-X.

URL http://wwW.sciencedirect.com/science/article/pii/ $037596018390097 \mathrm{X}$

[43] F. R. Graziani, V. S. Batista, L. X. Benedict, J. I. Castor, H. Chen, S. N. Chen, C. A. Fichtl, J. N. Glosli, P. E. Grabowski, A. T. Graf, S. P. Hau-Riege, A. U. Hazi, S. A. Khairallah, L. Krauss, A. B. Langdon, R. A. London, A. Markmann, M. S. Murillo, D. F. Richards, H. A. Scott, R. Shepherd, L. G. Stanton, F. H. Streitz, M. P. Surh, J. C. Weisheit, H. D. Whitley, Large-scale molecular dynamics simulations of dense plasmas: The cimarron project, High Energy Density Physics 8 (1) (2012) 105 - 131. doi:http://dx.doi.org/10.1016/j.hedp.2011.06.010.

URL http://www.sciencedirect.com/science/article/pii/ S1574181811000826

[44] P. Helander, M. Lisak, D. D. Ryutov, Formation of hot ion populations in fusion plasmas by close collisions with fast particles, Plasma Physics and Controlled Fusion 35 (3) (1993) 363.

URL http: //stacks . iop .org/0741-3335/35/i=3/a=006

[45] L. X. Benedict, J. N. Glosli, D. F. Richards, F. H. Streitz, S. P. Hau-Riege, R. A. London, F. R. Graziani, M. S. Murillo, J. F. Benage, Molecular dynamics simulations of electron-ion temperature equilibration in an $\mathbf{S f}_{6}$ plasma, Phys. Rev. Lett. 102 (2009) 205004. doi:10.1103/PhysRevLett.102.205004. URL http://link.aps .org/doi/10.1103/PhysRevLett.102.205004

[46] G. Kelbg, Theorie des quanten-plasmas, Annalen der Physik 467 (3-4) (1963) 219-224. doi:10.1002/andp.19634670308.

URL http://dx.doi.org/10.1002/andp.19634670308

[47] H. Minoo, M. M. Gombert, C. Deutsch, Temperature-dependent coulomb interactions in hydrogenic systems, Phys. Rev. A 23 (1981) 924-943. doi: 10.1103/PhysRevA.23.924.

URL http://link.aps.org/doi/10.1103/PhysRevA.23.924 
[48] S. T. Perkins, D. E. Cullen, Elastic nuclear plus interference cross sections for light charged particles, Nuclear Science and Engineering 77:1 (1981) 20-39.

[49] A. D. Fokker, Die mittlere energie rotierender elektrischer dipole im strahlungsfeld, Annalen der Physik 348 (5) (1914) 810-820. doi:10.1002/andp. 19143480507.

URL http://dx.doi.org/10.1002/andp.19143480507

[50] M. N. Rosenbluth, W. M. MacDonald, D. L. Judd, Fokker-Planck Equation for an Inverse-Square Force, Physical Review 107 (1) (1957) 1-6. doi:10.1103/PhysRev.107.1.

URL http://link.aps.org/doi/10.1103/PhysRev.107.1http://prola. aps.org/abstract/PR/v107/i1/p1_1

[51] A. Lenard, On Bogoliubov's kinetic equation for a spatially homogeneous plasma, Annals of Physics 10 (3) (1960) 390-400. doi: 10.1016/0003-4916(60)90003-8.

URL http://www.sciencedirect.com/science/article/pii/ 0003491660900038

[52] R. Balescu, Irreversible Processes in Ionized Gases, Physics of Fluids 3 (1960) (1960) 52. doi:10.1063/1.1706002.

URL http://link . aip.org/link/PFLDAS/v3/i1/p52/s1\&Agg=doi

[53] A. E. Turrell, M. Sherlock, S. J. Rose, A Monte Carlo algorithm for degenerate plasmas, Journal of Computational Physics 249 (0) (2013) $13-21$. doi:10.1016/j.jcp.2013.03.052.

URL http://wWw.sciencedirect.com/science/article/pii/ S0021999113002362

[54] L. L. Carter, E. D. Cashwell, Particle-Transport Simulation with the Monte Carlo Method, Technical Information Centre Energy Research and Development Administration, 1975.

[55] I. Lux, L. Koblinger, Monte Carlo Particle Transport Methods: Neutron and Photon Calculations, CRC press, Boca Raton, 1991.

[56] O. L. Landen, R. Benedetti, D. Bleuel, T. R. Boehly, D. K. Bradley, J. A. Caggiano, D. A. Callahan, P. M. Celliers, C. J. Cerjan, D. Clark, G. W. Collins, E. L. Dewald, S. N. Dixit, T. Doeppner, D. Edgell, J. Eggert, D. Farley, J. A. Frenje, V. Glebov, S. M. Glenn, S. H. Glenzer, S. W. Haan, A. Hamza, B. A. 
Hammel, C. A. Haynam, J. H. Hammer, R. F. Heeter, H. W. Herrmann, D. G. Hicks, D. E. Hinkel, N. Izumi, M. G. Johnson, O. S. Jones, D. H. Kalantar, R. L. Kauffman, J. D. Kilkenny, J. L. Kline, J. P. Knauer, J. A. Koch, G. A. Kyrala, K. LaFortune, T. Ma, A. J. Mackinnon, A. J. Macphee, E. Mapoles, J. L. Milovich, J. D. Moody, N. B. Meezan, P. Michel, A. S. Moore, D. H. Munro, A. Nikroo, R. E. Olson, K. Opachich, A. Pak, T. Parham, P. Patel, H.-S. Park, R. P. Petrasso, J. Ralph, S. P. Regan, B. A. Remington, H. G. Rinderknecht, H. F. Robey, M. D. Rosen, J. S. Ross, J. D. Salmonson, T. C. Sangster, M. B. Schneider, V. Smalyuk, B. K. Spears, P. T. Springer, L. J. Suter, C. A. Thomas, R. P. J. Town, S. V. Weber, P. J. Wegner, D. C. Wilson, K. Widmann, C. Yeamans, A. Zylstra, M. J. Edwards, J. D. Lindl, L. J. Atherton, W. W. Hsing, B. J. MacGowan, B. M. V. Wonterghem, E. I. Moses, Progress in the indirectdrive national ignition campaign, Plasma Physics and Controlled Fusion 54 (12) (2012) 124026.

URL http: //stacks . iop.org/0741-3335/54/i=12/a=124026

[57] B. Appelbe, J. Chittenden, The production spectrum in fusion plasmas, Plasma Physics and Controlled Fusion 53 (4) (2011) 045002.

URL http: //stacks . iop .org/0741-3335/53/i=4/a=045002

[58] J. A. Frenje, C. K. Li, F. H. Seguin, D. T. Casey, R. D. Petrasso, D. P. McNabb, P. Navratil, S. Quaglioni, T. C. Sangster, V. Y. Glebov, D. D. Meyerhofer, Measurements of the differential cross sections for the elastic $n-{ }^{3} \mathrm{H}$ and $n{ }^{2} \mathrm{H}$ scattering at $14.1 \mathrm{mev}$ by using an inertial confinement fusion facility, Phys. Rev. Lett. 107 (2011) 122502. doi:10.1103/PhysRevLett.107.122502. URL http://link . aps .org/doi/10.1103/PhysRevLett.107.122502 